\title{
Etude préliminaire de l'innocuité et du pouvoir immunogène de la souche atténuée VD $47 / 25$ de camelpoxvirus
}

\author{
Nguyen-Ba-Vy ${ }^{*}$ L. Guerre ${ }^{2 * *}$ G. Saint-Martin $1^{\text {1*** }}$
}

Mots-clés

Camelus dromedarius - Dromadaire Poxviridae - Vaccin - Anticorps Mauritanie.
Résumé

L'examen de l'innocuité et du pouvoir immunogène de la souche atténuée de camelpoxvirus $V D_{47 / 25}$ a été effectué sur 30 chamelons en Mauritanie. Aucun signe clinique n'a été constaté durant les 40 jours d'observation suivant la vaccination. Les titres des anticorps neutralisants des sérums prélevés durant cette période sont restés faibles (1/4-1/16). Les titrages in vivo d'une souche virulente sur des vaccinés et des témoins ont permis le calcul de la dose protectrice 50 p. $100\left(\mathrm{DP}_{50}\right)$ qui renfermait l'équivalent de $10^{3,7} \mathrm{DICT}_{50}$ (dose infectant 50 p. 100 des cultures cellulaires). D'autres études seront nécessaires pour la détermination de la dose vaccinale protégeant 95 p. 100 des vaccinés.

\section{INTRO DUCTION}

L'élevage de dromadaires se développe de plus en plus dans les pays africains, au Moyen-Orient et en Asie centrale, non seulement à cause des traditions socio-culturelles, mais aussi pour des raisons économiques. Celles-ci tiennent à une meilleure pérennité du capital de production que représentent les troupeaux, et à la bonne rentabilité de son exploitation commerciale, qui incitent davantage de commerçants et d'agriculteurs à investir dans cet élevage, face aux demandes accrues en lait et en viande (17).

Ce développement a pour corollaire des problèmes d'alimentation et de pathologie $(3,6,7)$. Les dromadaires sont réputés plus résistants aux maladies que les autres espèces domestiques, mais certaines enzooties comme la trypanosomose, la variole et l'ecthyma contagieux affectent actuellement des troupeaux de camélidés en créant des pertes économiques considérables $(1,8,9,17)$. La variole des dromadaires peut causer la mort des jeunes, mais aussi des adultes lorsqu'ils ont été mal nourris et affaiblis par une infection latente ou concomitante due à d'autres agents pathogènes.

La prévention de cette maladie, selon la méthode traditionnelle (inoculation d'un prélèvement d'animal malade à un animal sain après une atténuation par des procédés empiriques) est très risquée, tandis que l'usage du virus de la vaccine (8) pour obtenir une immunité croisée, a été abandonné suite aux recommandations de l'OMS en 1977, après l'éradication de la variole humaine. Certains vaccins homologues anti-camelpox ont été essayés par

1*. CIRAD-EMVT, Campus international de Baillarguet, BP 5035 34032 Montpellier Cedex, France

2. CNERV, BP 167, Nouakchott, République islamique de Mauritanie

* Adresse actuelle

** Adresse actuelle : LANAVET, BP 503, Garoua, Cameroun

*** Adresse actuelle : Ministère de l'éducation nationale, de l'enseignement supérieur et de la recherche, Mission scientifique et technique, DSPT9, 77 avenue Denfert-Rochereau, 75014 Paris, France différents auteurs $(5,10)$. Les résultats d'une étude préliminaire de l'innocuité et du pouvoir immunogène de l'une des souches atténuées de camelpoxvirus sont exposés dans ce travail.

\section{MATERIEL ET METHODES}

\section{Souches de camelpoxvirus}

Souche $\mathrm{VD}_{47 / 25}$

C'est une souche atténuée dérivant de la souche virulente $\mathrm{VD}_{47}$ isolée des dromadaires du Niger en 1981 (12). Elle a subi 65 subcultures sur des cellules $\mathrm{IB}-\mathrm{RS}_{2}$ à $36^{\circ} \mathrm{C}$ et 18 passages à $25^{\circ} \mathrm{C}$ avant d'être utilisée pour la fabrication du vaccin sur des cellules Vero.

\section{Souche VDR-A}

La souche virulente VDR-A 2 a été isolée par Lejan en 1987 sur des dromadaires de Mauritanie. Elle se multiplie bien sur des cellules d'explantation de dromadaires ou de moutons et s'adapte facilement aux cellules Vero.

\section{Production du vaccin brut}

Après la destruction de la nappe des cellules Vero par le camelpoxvirus, la culture a été soumise à cinq cycles de congélation-décongélation, puis à une centrifugation à $1000 \mathrm{~g}$ pendant $15 \mathrm{~min}$. Le surnageant constituait le vaccin brut qui peut être conservé à $-80^{\circ} \mathrm{C}$ ou lyophilisé après vérification de l'absence de toute contamination.

\section{Sélection des chamelons sensibles}

Il n'est pas facile de trouver en Mauritanie, où sévit la variole des dromadaires à l'état endémique, des chamelons parfaitement sensibles, car la majorité d'entre eux possèdent un certain degré de résistance au camelpoxvirus. Elle est due à la persistance des 
anticorps maternels transmis avec le colostrum ou à la suite d'une infection naturelle bénigne. L'absence des anticorps neutralisants ne signifie pas toujours une sensibilité de l'animal car la résistance contre ce virus est basée non seulement sur des anticorps neutralisants, mais aussi sur une immunité à médiation cellulaire. Les tests d'examen de ce dernier type d'immunité n'étaient pas praticables sur le terrain, il a donc été décidé de sélectionner parmi les chamelons de 3 à 18 mois dans différents élevages aux alentours de Nouakchott, 35 sujets ayant des titres d'anticorps neutralisants inférieurs à $1 / 4$, dans l'espoir de trouver un certain nombre d'animaux sensibles pour les différents lots. Ceux-ci ont été enfermés simplement dans des enclos à l'air libre.

\section{Vaccination des chamelons et test d'innocuité}

Une suspension du virus $\mathrm{VD}_{47 / 25}$ contenant $10^{5} \mathrm{DICT}_{50} / \mathrm{ml}$ (dose infectant 50 p. 100 des cultures cellulaires) et ses dilutions de $10^{-1}$ à $10^{-5}$ ont été inoculées à la dose de $5 \mathrm{ml}$ en sous-cutané à 6 lots de chamelons. Chaque sujet a donc reçu respectivement de $10^{0,7}$ à $10^{5,7} \mathrm{DICT}_{50}$. Des injections de plus grand volume, $50 \mathrm{ml}$ d'une suspension de virus $\mathrm{VD}_{47 / 25}$ contenant $10^{5} \mathrm{DICT}_{50}$, soit l'équivalent de $10^{6,7} \mathrm{DICT}_{50}$, ont été faites sur deux chamelons pour tester l'innocuité de la préparation.

Des échantillons de sang ont été prélevés juste avant la vaccination (le jour même) et chaque semaine suivante durant une période de 40 jours, au niveau de la partie haute de la veine jugulaire à l'aide de tubes vacutainer de $10 \mathrm{ml}$, munis de gel séparateur de sérum et d'activateur de coagulation (Becton-Dickinson SST). Les sérums exsudés ont été décantés dans des tubes stériles et gardés au congélateur jusqu'aux titrages.

\section{Titrage des anticorps neutralisants}

La recherche des anticorps neutralisants a été effectuée par la microméthode de séroréduction de l'effet cytopathique (SRECP) du virus sur des plaques à 96 cupules à fond plat. Pour cela, un stock de virus $\mathrm{VD}_{47 / 25}$ a été conservé à $-80^{\circ} \mathrm{C}$ dans des cryotubes. Un premier titrage de cette suspension virale sur des plaques de cellules d'explantation ou de cellules Vero a permis de trouver la dilution correspondant à $100 \mathrm{DICT}_{50}$ dans $25 \mu \mathrm{l}$. Un titrage secondaire à partir de ce niveau et avec les dilutions selon la progression géométrique de raison 2 , a mis en évidence la dilution optimale qui était la dose minimale de virus capable de détruire 80 p. 100 de la surface de la nappe cellulaire dans un délai de 8 jours et dans 5 cupules en même temps.

Les sérums à examiner ont été décomplémentés puis dilués avec une suspension optimale de virus selon la progression géométrique de raison 2. Ces mélanges ont été incubés pendant $1 \mathrm{~h}$ à $37^{\circ} \mathrm{C}$ puis 1 nuit à $8^{\circ} \mathrm{C}$ avant leur distribution sur une plaque de cellules de $20 \mathrm{~h}$, à raison de $25 \mu \mathrm{l}$ par cupule et dans 5 cupules par dilution. Après une nouvelle incubation dans une étuve à $\mathrm{CO}_{2}$ à $37^{\circ} \mathrm{C}$ pendant $1 \mathrm{~h}$, chaque cupule a reçu $150 \mu \mathrm{l}$ de milieu d'entretien (MEM/Earle additionné de 5 p. 100 de sérum).

La lecture a été effectuée au $8^{\mathrm{e}}$ jour d'incubation à $37^{\circ} \mathrm{C}$. Le titre sérique, calculé selon la méthode de Reed-Muench (16), était le $\log _{10}$ de l'inverse de la dilution du sérum. La moyenne géométrique était la moyenne des $\log _{10}$ des titres d'un groupe.

\section{Epreuve des animaux vaccinés en Mauritanie}

Quarante jours après la vaccination, les chamelons ont été soumis, à une épreuve avec la souche virulente VDR-A $A_{2}$, qui était en fait titrée sur les vaccinés et les témoins. Les différentes dilutions virales ont été inoculées en intradermique sur un côté rasé du thorax à raison de $0,25 \mathrm{ml}$ par point et sur 5 points par dilution. La lecture était effectuée respectivement au $5^{\mathrm{e}}, 9^{\mathrm{e}}$ et $12^{\mathrm{e}}$ jours après l'inoculation et les titres du virus virulent étaient calculés selon la méthode de Reed-Muench. La date, la durée et le degré des réactions cutanées étaient notés ainsi que des signes de morbidité ou des cas de mortalité. En général, les réactions cutanées étaient absentes ou disparaissaient rapidement chez les animaux bien immunisés. L'indice de protection (ID) était la différence entre les titres moyens (en $\log _{10}$ ) du virus virulent trouvé respectivement sur le lot témoin d'une part, et sur le lot vacciné d'autre part. La dose protectrice 50 p. $100\left(\mathrm{DP}_{50}\right)$ a été calculée selon la méthode de Spearman-Karber (4).

\section{RESU LTATS}

\section{Innocuité de la souche atténuée VD $D_{47 / 25}$ de camelpoxvirus}

Sur deux chamelons ayant reçu en sous-cutané $50 \mathrm{ml}$ de la suspension de virus $\mathrm{VD}_{47 / 25}$ contenant $10^{5} \mathrm{DICT}_{50} / \mathrm{ml}$, aucune réaction cutanée (ni érythème, ni nodule ni pustule), ni aucun signe clinique n'ont été observés durant les 25 jours de surveillance.

La vaccination de 28 autres chamelons avec des doses allant de $10^{0,7}$ à $10^{5,7} \mathrm{DICT}_{50}$, sous volume de $5 \mathrm{ml}$ en sous-cutané, n'a provoqué aucun symptôme de variole sur tous ces animaux durant les 40 jours d'observation.

Ces résultats ont permis d'affirmer que la souche atténuée $\mathrm{VD}_{47 / 25}$ n'était pas pathogène pour les chamelons de Mauritanie.

\section{Pouvoir immunogène de la souche de virus $V D_{47 / 25}$}

\section{Examen des anticorps neutralisants}

L'examen des sérums prélevés juste avant la vaccination a révélé des titres très faibles ou négligeables pour la plupart d'entre eux. Les moyennes géométriques dans les différents lots étaient inférieures à 0,7 (en $\left.\log _{10}\right)$ malgré l'existence de quelques titres individuels plus élevés (tableaux I et I bis).

Après la vaccination, les titres sont aussi restés relativement faibles durant 40 jours, avec des moyennes géométriques variant de 0,6 à 1,08. Les deux premiers lots avaient respectivement des titres moyens de 1,08 et 0,98 au $21^{\mathrm{e}}$ jour. Quelques titres individuels se sont élevés jusqu'à 1,2 (tableaux I et I bis).

Après l'épreuve avec la souche virulente VDR-A $A_{2}$, une très nette augmentation des titres d'anticorps neutralisants a été constatée au 13 e jour sur tous les chamelons, avec des moyennes de 2 à 2,4 (en $\left.\log _{10}\right)$.

\section{Examen des titres du virus virulent sur des animaux vaccinés et des} témoins

La majorité des chamelons en Mauritanie où la variole des dromadaires existe à l'état endémique, possèdent un certain degré de résistance à cette maladie. C'est pourquoi après la comparaison des titres du virus virulent sur des témoins et des vaccinés, il a été décidé de corriger ce biais en prenant la valeur de 1,50 (en $\log _{10}$ ) comme seuil de protection : un animal qui permettait au virus virulent d'afficher des titres égaux ou supérieurs à 1,50 n'était pas considéré comme protégé.

Dans le premier lot, 5 chamelons ont reçu du vaccin non dilué, soit $10^{5,7} \mathrm{DICT}_{50}$ pour chacun. Lors de l'épreuve, les titres du virus virulent sont restés inférieurs à 1,50 au $12^{\mathrm{e}}$ jour chez 4 sujets qui n'ont montré d'ailleurs aucun signe clinique (tableau I). Le 5e animal avec des lésions buccales discrètes au $12^{\mathrm{e}}$ jour, a révélé un 


\section{Tableau I}

Titres des anticorps neutralisants après la vaccination et titres du virus virulent sur des chamelons vaccinés

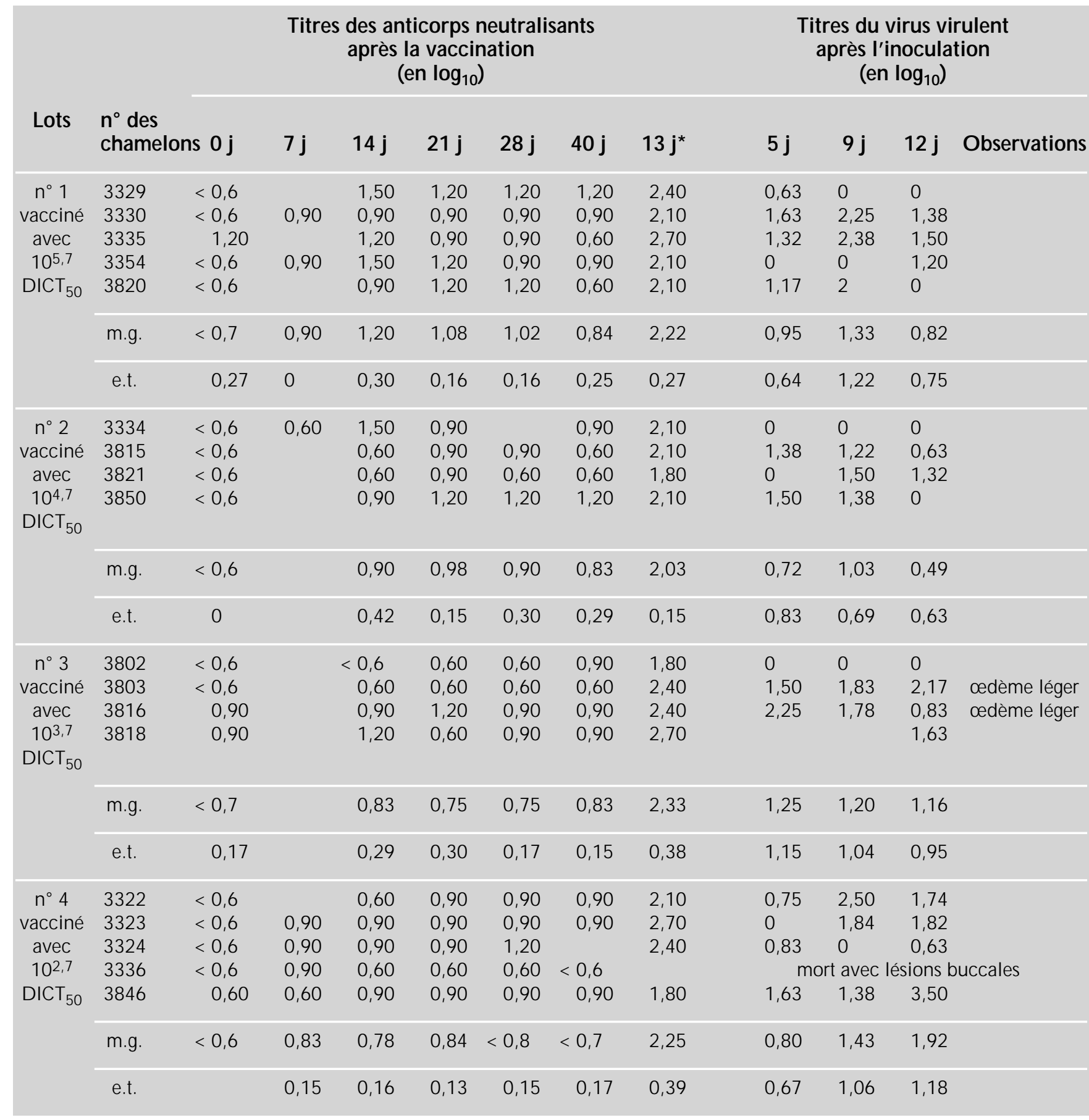

* après l'épreuve ; m.g. : moyenne géométrique ; e.t. : écart-type

titre de 1,50 ; ses anticorps préexistants auraient partiellement neutralisé l'effet du vaccin. Le titre moyen trouvé sur ce lot était 0,82 $\left(\log _{10}\right)$.

Dans le deuxième lot, 4 chamelons ayant reçu chacun $10^{4,7}$ DICT $_{50}$ du vaccin n'ont permis au virus virulent d'afficher que des titres inférieurs à 1,50 au 12e jour de l'épreuve (tableau I), avec une moyenne de 0,49 et une absence totale de tout symptôme caractéristique.
Dans le troisième lot, chacun des 4 chamelons a été vacciné avec $10^{3,7} \mathrm{DICT}_{50}$. Après l'épreuve, seulement 2 d'entre eux étaient considérés comme protégés en montrant au $12^{\mathrm{e}}$ jour des titres du virus virulent inférieurs à 1,50 (tableau I). Le titre viral moyen était 1,16 .

Dans le quatrième lot, le vaccin, à la dose individuelle de $10^{2,7}$ $\mathrm{DICT}_{50}$ a été administré à 5 chamelons. Les titrages in vivo du virus virulent y ont donné des valeurs supérieures à 1,50 , allant de 


\section{Tableau I bis}

Titres des anticorps neutralisants après la vaccination et titres du virus virulent sur des chamelons vaccinés et des témoins

\section{Titres des anticorps neutralisants après la vaccination \\ $\left(\right.$ en $\left.\log _{10}\right)$}

\section{Titres du virus virulent après l'inoculation $\left(\right.$ en $\left.\log _{10}\right)$}

Lots $\quad n^{\circ}$ des chamelons

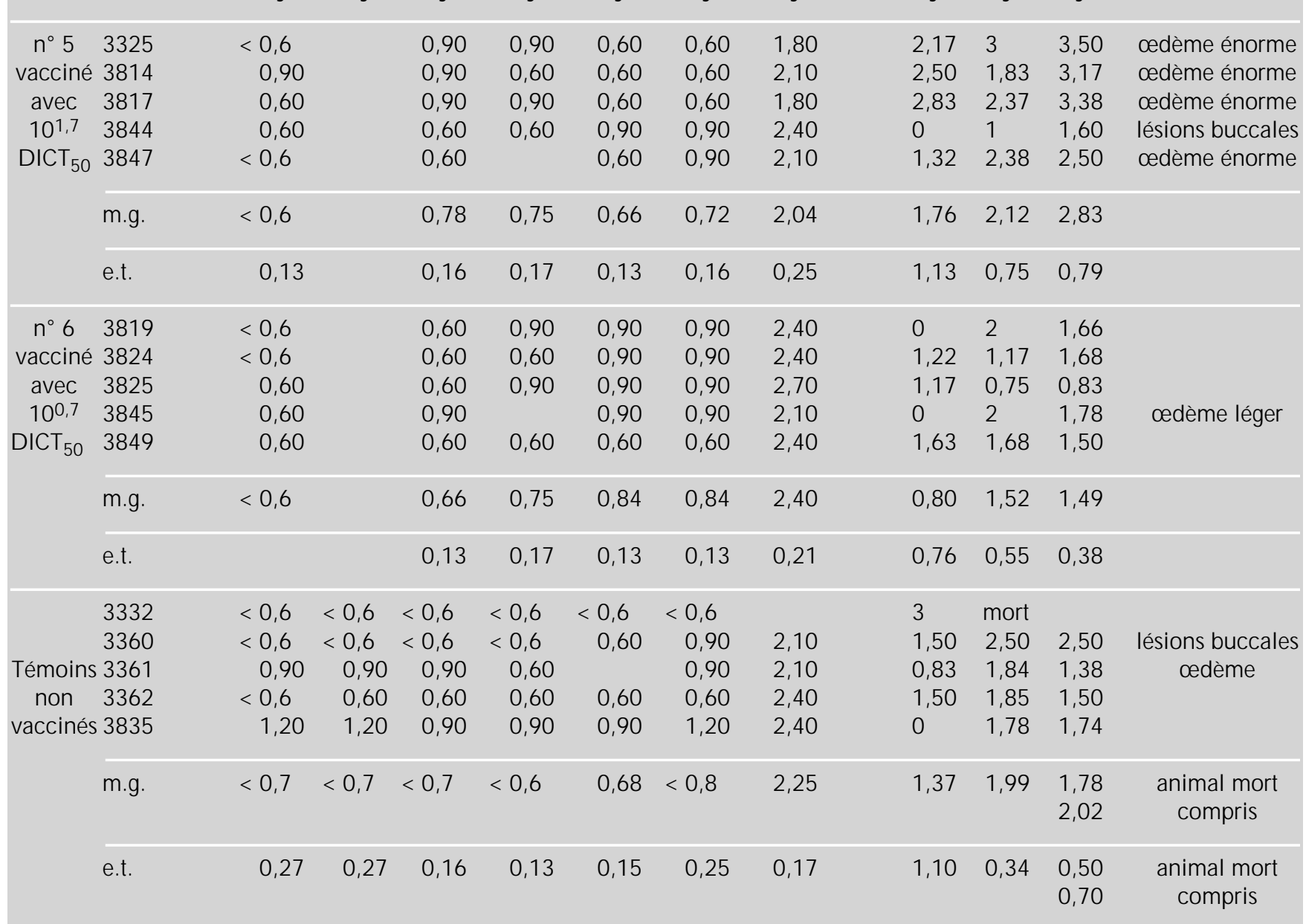

* après l'épreuve ; m.g. : moyenne géométrique ; e.t. : écart-type

1,74 à 3,50, sur 4 sujets dont l'un est mort avec des lésions buccales caractéristiques (tableau I). Le $5^{\mathrm{e}}$, avec un titre viral de 0,63, a été considéré comme immunisé. Ce lot a montré un titre moyen de 1,92 au $12^{\mathrm{e}}$ jour.

Dans le cinquième lot, aucun des 5 sujets vaccinés avec $10^{1,7}$ $\mathrm{DICT}_{50}$ ne pouvait être considéré comme protégé, puisqu'au $12^{\mathrm{e}}$ jour de l'épreuve tous les titres du virus virulent se sont élevés audessus de 1,50 avec une variation de 1,60 à 3,50 (tableau I bis) et avec une moyenne de 2,83, après l'apparition des odèmes importants et des lésions buccales.

Dans le sixième lot, la vaccination de 5 chamelons avec $10^{0,7}$ $\mathrm{DICT}_{50}$, n'a pas conféré une immunité valable à 4 d'entre eux dont les titres du virus virulent au $12^{\mathrm{e}}$ jour de l'épreuve se sont révélés égaux ou supérieurs à 1,50 (tableau I bis). Une moyenne de 1,49 a été trouvée sur ce lot.
Dans le lot des témoins, les 5 animaux non vaccinés ont montré qu'ils n'étaient pas très sensibles au virus virulent en affichant des titres allant de 1,38 à 3 avec une moyenne de 2,02 (tableau I bis). Si cette dernière valeur avait été utilisée comme seuil de protection, 100 p. 100 de protégés aurait été trouvés dans les deux premiers lots, 75 p. 100 dans le troisième, 60 p. 100 dans le quatrième, 20 p. 100 dans le cinquième, mais aussi 100 p. 100 dans le sixième lot et 60 p. 100 dans le groupe des témoins, ce qui aurait rendu les résultats aberrants et inexploitables. C'est pourquoi il a été décidé d'y apporter des corrections, en choisissant comme seuil de protection la valeur de 1,50 qui correspondait à la moyenne $(1,49)$ des titres virulents trouvés sur des chamelons du sixième lot. Les indices de protection sont ainsi devenus plus cohérents avec des valeurs respectives de 0,7 pour le premier lot, 1 pour le deuxième, 0,34 pour le troisième, et négatif pour tous les autres lots, y compris le lot témoin. 


\section{Détermination de la $\mathrm{DP}_{50}$ (dose protégeant 50 p. 100 des vaccinés)}

Les résultats des titrages du virus virulent VDR-A sur des chamelons vaccinés ont permis la détermination du nombre des animaux protégés dans chaque lot (tableau II). Après une correction selon les valeurs des lots voisins, tous les sujets vaccinés avec $10^{5,7}$ $\mathrm{DICT}_{50}$ ont pu être considérés comme protégés puisque tous ceux qui avaient reçu la dose de $10^{4,7}$ étaient immunisés. Tous les animaux du lot témoin et du lot vacciné avec $10^{0,7} \mathrm{DICT}_{50}$ ont été estimés comme non-protégés, car tous les sujets vaccinés avec $10^{1,7}$ DICT $_{50}$ sont restés sensibles. La moitié des chamelons vaccinés avec $10^{3,7} \mathrm{DICT}_{50}$ sont devenus résistants (2/4) ainsi que l'un des 5 vaccinés avec $10^{2,7} \mathrm{DICT}_{50}$. La dose protectrice 50 p. $100\left(\mathrm{DP}_{50}\right)$ calculée selon la méthode de Spearman-Karber, correspondait à $10^{3,7} \mathrm{DICT}_{50}$ du virus $\mathrm{VD}_{47 / 25}$.

\section{Détermination de la dose vaccinale}

Une $\mathrm{DP}_{50}$ de $10^{3,7} \mathrm{DICT}_{50}$ ne protégeait qu'environ 50 p. 100 des vaccinés. La valeur de la dose vaccinale qui immunisait 95 p. 100 des sujets n'est pas encore bien déterminée. Néanmoins, les résultats des titrages de la souche virulente sur des chamelons vaccinés, ont permis d'estimer qu'elle se situe aux alentours de $10^{4,7}$ DICT $_{50}$. D'autres travaux d'expérimentation seront encore nécessaires pour déterminer cette valeur optimale.

\section{DISCUSSION}

L'immunité contre la variole des dromadaires est assurée à la fois par des anticorps circulants et par des mécanismes à médiation cellulaire. La présence des anticorps neutralisants à de faibles niveaux (1/4-1/8) a été constatée aussi bien chez les animaux sensibles que chez les résistants ; seuls des titres élevés (> 1/32-1/64) ont une certaine corrélation avec l'état immunitaire de l'animal.

La détection des chamelons sensibles avant l'expérimentation est une opération très délicate. Cette sensibilité varie beaucoup selon l'individu, notamment parmi les animaux en provenance des régions où la variole sévit à l'état endémique. Certains peuvent acquérir une immunité passive par l'intermédiaire du colostrum maternel ou une immunité active après une infection naturelle bénigne. La seule vérification de l'absence des anticorps neutrali- sants s'est révélée insuffisante pour déterminer l'état de sensibilité d'un animal. Il existe des méthodes plus efficaces pour évaluer une immunité à médiation cellulaire, comme le test de migration des leucocytes $(15,18)$ basé sur la propriété des lymphocytes sensibilisés par les antigènes, de sécréter lors d'un nouveau contact avec ceux-ci, des lymphokines inhibant la migration spontanée des leucocytes (macrophages ou polynucléaires). Le test de transformation lymphoblastique $(2,13,19)$ permet aussi la détection des lymphocytes spécifiquement fonctionnels pour un antigène déterminé, qui réagissent à un nouveau contact par la production des lymphokines mitogènes : un indicateur oxydo-redox fluorescent (Alamar Blue) (14) facilite la mesure de la croissance cellulaire. L'examen du taux des lymphocytes cytotoxiques y est aussi d'une grande utilité. La mise en œuvre de tous ces tests, si elle ne pose pas de problèmes pour un laboratoire bien équipé et pourvu en personnel compétent, s'est révélée irréalisable sur le terrain, lors des opérations de sélection sur un grand nombre d'animaux. Les auteurs n'ont donc utilisé en Mauritanie que le test de séroréduction de l'effet cytopathique pour trouver des chamelons sensibles. L'interprétation des résultats obtenus sur de tels animaux devait tenir compte des biais en apportant des corrections adéquates. Les auteurs pensent que ces résultats se rapprochent plus de la réalité écopathologique du pays que ceux obtenus sur des animaux parfaitement sensibles, en provenance des continents indemnes de la variole des dromadaires.

\section{CONCLUSION}

La souche atténuée $\mathrm{VD}_{47 / 25}$ de camelpoxvirus n'est pas pathogène pour les chamelons de Mauritanie. Elle possède un pouvoir immunogène avec une $\mathrm{DP}_{50}$ équivalente à $10^{3,7} \mathrm{DICT}_{50}$. La dose vaccinale protégeant 95 p. 100 des vaccinés, encore mal déterminée, devra faire l'objet d'une autre étude.

\section{Remerciements}

Nos remerciements sont adressés au Dr Diallo Boubacar Cissé, Directeur du Centre national de l'élevage et de recherches vétérinaires à Nouakchott (République islamique de Mauritanie) qui nous a accordé toutes les facilités pour la réalisation de cette expérimentation, au Dr Mulato qui nous a procuré une aide précieuse par sa connaissance du terrain et de l'élevage des dromadaires et à

\section{Tableau II}

Détermination de la $D P_{50}$ du vaccin $V D_{47 / 25}$

\begin{tabular}{|c|c|c|c|c|c|c|c|}
\hline $\mathrm{N}^{\circ}$ des lots & 1 & 2 & 3 & 4 & 5 & 6 & Témoins \\
\hline $\begin{array}{l}\text { Doses de virus VD } \\
\text { pour chaque chamelon } \\
\left.\text { (DICT } 50 \text { en } \log _{10}\right)\end{array}$ & 5,7 & 4,7 & 3,7 & 2,7 & 1,7 & 0,7 & \\
\hline Nombre d'animaux utilisés & 5 & 4 & 4 & 5 & 5 & 5 & 5 \\
\hline Nombre d'animaux protégés & 4 & 4 & 2 & 1 & 0 & 1 & 1 \\
\hline Nombre corrigé & 5 & 4 & 2 & 1 & 0 & 0 & 0 \\
\hline Pourcentage & 100 & 100 & 50 & 20 & 0 & 0 & 0 \\
\hline $\begin{array}{l}\text { Doses de virus VD } \\
\text { dans } 1 \mathrm{DP}_{50} \\
\left.\text { (DICT }_{50} \text { en } \log _{10}\right)\end{array}$ & $10^{3,7}$ & & & & & & \\
\hline
\end{tabular}


Mme Denise Boudal pour sa collaboration technique. Nous rendons également hommage à $\mathrm{M}$. Thiémoko Cissé qui a apporté ses compétences techniques à ces travaux et dont nous regrettons aujourd'hui la disparition.

Les travaux en Mauritanie ont été financés par le Ministère français de la coopération à travers l'Unité de coordination pour l'élevage camelin (UCEC).

\section{BIBLIOGRAPHIE}

1. DAVIES F.G., MBUGUA H., OTEMA C., WILSON A., 1985. The prevalence of antibody to camelpoxvirus in six different herds in Kenya. J. comp. Path., 95: 633-635.

2. DUTTON R.W., EADY J.D., 1964. An in vitro system for the study of the mechanism of antigenic stimulation in the secondary response. Immunology , 7: 40-53.

3. FASSI-FEHRI M.M., 1987. Les maladies des camélidés. Rev. sci. tech. O ff. int. Epiz., 6 (2): 315-335.

4. FINNEY J.D., 1964. Statistical method in biological assay, 2nd ed. London, U.K., Griffin.

5. HAFEZ S.M., AL-SUKAYRAN A., DELA CRUZ D., MAZLOUM K.S. AL-BO KMY A.M., AL-MUKAYEL A., AMJAD A.M., 1992. Development of a live cell culture camelpox vaccine. Vaccine, 10 (8): 533-539.

6. HEDGER R.S., BARNETT I.T.R., GRAY D.F., 1980. Some virus diseases of domestic animals in the Sultanate of 0 man. Trop. Anim. Health Prod., 12: 107-114.

7. HIGGINS A.J., 1983. Observation on the disease of Arabian camels (Camelus dromedarius) and their control. A review. Commw. Bur. Anim. Health vet. Bull., 53 (12): 1089-1097.

\section{Summary}

N guyen-Ba-Vy, G uerre L., Saint-Martin G. Preliminary study of the safety and immunogenicity of the attenuated VD $V_{47 / 25}$ strain of camelpoxvirus

The safety and immunogenicity of the attenuated VD $47 / 25$ strain of camelpoxvirus were tested on 30 camel calves in Mauritania. Post-inoculation clinical symptoms were absent during the 40 days of observation. Serum samples collected during this period showed low levels of neutralizing antibodies (1/4-1/16). In vivo titration of a virulent strain of camelpoxvirus in vaccinated camels and control animals enabled the calculation of the $\mathrm{PD}_{50}$ (50\% protective dose) which contained the equivalent of $10^{3.7} \mathrm{TCID}_{50}(50 \%$ cell culture infective dose). 0 ther studies are still required to determine the dose of this vaccine needed to protect $95 \%$ of vaccinated animals.

Key words : Camelus dromedarius - Dromedary - Poxviridae Vaccine - Antibody - Mauritania.
8. HIGGINS A.J., SILVEY R.E., ABDELGRAFIR A.E., KITCHING R.P. 1992. The epidemiology and control of an outbreak of camel pox in Bahrain. In: Proc. 1st Int. Camel Conf., Dubai, UAE, 2nd-6th February, p.101-104.

9. JEZEK Z., KRIZ B., ROTHBAUER V., 1983. Camelpox and its risk to the human population. J. Hyg. Epidemiol. Microbiol. Immunol., 27: 29.

10. KAADEN O.R., WALZ A., CZERNY C.P., WERNERY U., 1992. Progress in the development of a camel pox vaccine. In: Proc. 1st Int. Camel. Conf., Dubai, UAE, 2nd-6th February, p.47-49.

11. MUNZ E., 1992. Pox and pox like diseases in camels. In: Proc. 1st Int. Camel Conf., Dubai, UAE, 2nd-6th February, p.43-46.

12. NGUYEN-BA-VY, RICHARD D., GILLET J.P., 1989. Propriétés d'une souche d'orthopoxvirus isolée des dromadaires du Niger. Revue Élev. M éd. vét. Pays trop., 42 (1) : 19-25.

13. OPPEN HEIM J.J., SCHECTER B., 1976. Lymphocyte transformation. In: Rose N.E., Friedman $H_{\text {., }}$ eds, Manual of Clinical Immunology. W ashington, DC, USA, American Society of M icrobiology, p. 81-94.

14. PAGE B., PAGE M., NOEL C., 1993. A new fluorometric assay for cytotoxicity measurements in vitro. Int. J. O ncol., 3: 473-476.

15. PERSON J.M., LESOURD B., POIRIER J., EDIRNELIS A., MARESCOT M.R., THIOLLET M., MOULIAS R., PILET Ch., 1981. Production d'extraits dialysables leucocytaires chez les bovins. I. Protocole et contrôle de l'immunisation des animaux. Comp. Immunol. Microbiol. infect. Dis., 4 (1): 47-57.

16. REED I.J., MUENCH H., 1938. A simple method for estimating fifty per cent end points. Am. J. Hyg., 27: 493-497.

17. SAINT-MARTIN G., 1994. Le dromadaire, un allié dans le désert. BioFutur, 136: 20-24.

18. SPITLER L., HUBER H., FUDENBERG H.H., 1969. Inhibition of capillary migration by antigen-antibody complexes. J. Immunol., 102: 404-411.

19. WITTMAN G., BARBENBACH G., JAKUBIK J., 1976. Cell-mediated immunity in Aujeszky disease virus invected pigs. I. Lymphocyte stimulation. Arch. Virol., 50: 315-222.

Reçu le 30.5.95, accepté le 25.11.96

\section{Resumen}

N guyen-Ba-Vy, Guerre L., Saint-Martin G. Estudio preliminar sobre la inocuidad y el poder inmunógeno de la cepa atenuada $\mathrm{VD}_{47 / 25}$ de camelpoxvirus

Se llevó a cabo el examen de la inocuidad y del poder inmunógeno de la cepa atenuada de camelpoxvirus $\mathrm{VD}_{47 / 25}$, sobre 30 camellos jóvenes en Mauritania. Durante los 40 días de observación, post vacunación, no se observó ningún signo clínico. Los títulos de anticuerpos neutralizadores de los sueros extraídos durante este período se mantuvieron bajos (1/4-1/16). Las titulaciones in vivo de una cepa virulenta sobre animales vacunados y testigos, permitió el cálculo de la dosis protectora 50 p.100 ( $\left.\mathrm{DP}_{50}\right)$, equivalente a 103,7 DICT $_{50}$ (dosis infectante de 50 p. 100 de los cultivos celulares). O tros estudios serán necesarios para la determinación de la dosis vaccinal protectora de 95 p. 100 de los animales vacunados.

Palabras clave : Camelus dromedarius - Dromedario Poxviridae - Vacuna - Anticuerpo - M auritania. 